\title{
Universalism and Junk
}

\section{A. J. COTNOIR}

\begin{abstract}
Those who accept the necessity of mereological universalism face what has come to be known as the 'junk argument' due to Bohn (2009) and Schaffer (2010), which proceeds from (i) the incompatibility of junk with universalism and (ii) the possibility of junk, to conclude that mereological universalism isn't metaphysically necessary. Most attention has focused on (ii); however recent authors have cast doubt on (i). This paper undertakes a defense of premise (i) against three main objections. The first is a new objection to the effect that Bohn's (2009) defence of it presupposes far too much. In particular, Bohn's suppositions entail mereological extensionalism. I show that one can defend premise (i) from a much weaker set of assumptions. Hence variants of the junk argument can be shown to apply to non-extensional mereological systems as well. The second objection due to Contessa (2012) is that those who accept unrestricted composition should only accept the existence of binary sums (which are compatible with junk) rather than infinitary fusions. I argue this conception of unrestricted composition is problematic: it is in conflict with an intuitive remainder principle. The final objection is due to Spencer (2012). Spencer's view is that there is no absolutely unrestricted plural universal quantifier. So, any statement of the unrestricted fusion axiom will simply not rule out the existence of junky worlds. I argue that the failure of unrestricted quantification will not be enough by itself to establish the existence of junk. And furthermore, it is not clear whether this view counts as a form of mereological universalism. As a result, I suggest that if one wants to reject the junk argument, premise (ii) is the only viable option.
\end{abstract}

Keywords junk, mereology, universalism, extensionality, unrestricted quantification 
Universalism is the view that composition is unrestricted; that is, for any things whatsoever, there is a whole composed of them. A number of philosophers maintain that universalism is true, indeed that it is metaphysically necessary. ${ }^{1}$ But universalism is one of the most controversial doctrines of classical mereology. A standard objection is that universalism commits us to too many composite objects, including gerrymandered objects composed of say, the Eiffel Tower and the tip of your nose. These are not objects recognised in our common sense conception of the world.

A more recent objection, however, contends that universalism commits us to too few composite objects. It requires parthood chains to 'top out' at some largest object. But must there be such a largest object? Why couldn't it be that for every composed whole, there is another that contains it? An argument has been put forward that undermines the view that universalism is metaphysically necessary; the argument appeals to the possibility of junky worlds. Junky worlds (introduced by Schaffer [24]) are worlds where everything in that world is a proper part of something. ${ }^{2}$ The junk argument, due to Bohn $[\mathrm{I}, 2,3]$, proceeds as follows:

(i) If universalism necessarily true, then there can be no junky worlds.

(ii) Junky worlds are metaphysically possible.

(iii) Therefore, universalism is either metaphysically contingent or necessarily false.

Much attention has been devoted to premise (ii). Bohn [I] contends that junky worlds satisfy three criteria for metaphysical possibility: conceivability, advocacy, and consistency.

But if we can conceive of junky worlds, and several prominent philosophers have taken the idea seriously, and there are no logical contradictions lurking, then we are hard pressed to deny the mere possibility of the world being junky. ([I, p. 29])

\footnotetext{
${ }^{1}$ Lewis [19] Rea [2 I], Sider [25], Varzi [33], van Cleve [32], and many more.

${ }^{2}$ This is the converse of the notion of a gunky world in which everything in that world has something as a proper part.
} 
The case for conceivability involves thought experiments: one might imagine for example that a universe just like ours is contained as a particle in some much larger replica universe, which is itself merely a particle in another replica universe, and so on. Such a world would be junky. Leibniz [18] and Whitehead [38] both held that the actual world is junky. ${ }^{3}$ Moreover, there are clearly formal models of junky mereologies, as we shall see below, and so the idea is logically consistent.

Schaffer $[24$, p. 65] by contrast, thinks that junk is inconceivable; conceiving of a world as a world requires it to be viewed as a totality, or a whole. And any world that contained junk would be an entity that isn't a proper part of anything, per impossible. Watson [37] also takes up the case against conceivability by objecting to the specific thought experiments proposed. For the record, I do not think Bohn's three criteria are sufficient for (or even provide good evidence for) metaphysical possibility, but the matter is too complicated for discussion here.

In any case, I do not wish to challenge the case for premise (ii). More recently premise (i) has come under fire. Contessa [6] and Spencer [28] have each provided independent reasons that 'universalists' should dispatch the junk argument by rejecting premise (i). In this paper, I undertake a defence of premise (i) against a variety of objections.

In $₫$ I, I put forward a new objection to Bohn's defence of premise (i) — that it is not available to proponents of non-classical mereologies. In $\$ \$ 2-3$ I generalise the defence of premise (i) to hold in virtually any merelogy and based on virtually any definition of mereological fusion. I address unsupplemented mereologies in $\$ 2$ which explicitly reject Bohn’s key assumption. In $\$_{3} \mathrm{I}$ address mutual parts mereologists, who might accept counterfeit junk that satisfies the definition in letter, but not in spirit. A slight revision in the definition of junk reestablishes premise (i) (or a mild variant thereof). In $\S_{4}$ I defend premise (i) against Contessa’s objection; and in $\$_{5}$, I defend it against Spencer's objection.

These defences of premise (i) show that it is particularly resilient and can be based on extremely minimal assumptions. The upshot, then, is that anyone who wants to reject the junk argument

\footnotetext{
${ }^{3}$ See Bohn [I, p. 28] and Simons [26, p. 83] for relevant quotations.
} 
must do so by rejecting premise (ii) instead.

\section{Extensionality \& Junk}

In his argument for premise (i), Bohn relies on the following mereological assumption, where $<$ is proper parthood, $\leq$ is parthood (' $x \leq y$ ' is defined as ' $x<y \vee x=y$ '), and $\circ$ is mereological overlap (' $x \circ y$ ' is defined as ' $\exists z(z \leq x \wedge z \leq y)$ ').

Weak Supplementation $x<y \rightarrow \exists z(z<y \wedge \neg z \circ x)$

Weak supplementation says that if something has a proper part, it must have another part disjoint from the first. Recall, a junky world is one in which everything is a proper part of something:

Junk $\forall x \exists y(x<y)$

Bohn's argument for premise (i) is as follows:

[A]ssume universalism is true in all possible worlds and that some possible world $w$ is junky. Then universalism is true in $w$. Consider the plurality of everything there is in $w$, call it $a a$. By universal instantiation, $\exists y(a a$ compose $y)$; by existential instantiation, aa compose $U$. By definition [of junk], it is true in $w$ that $\forall x \exists y(x<y)$. By universal instantiation, $\exists y(U<y)$. But $U$ is composed of everything in $w$, and hence, by definition [of composition], everything in $w$ is a part of it, including itself. But then nothing can have $U$ as a proper part, because if so, by [weak] supplementation, there would be something that shares no part with $U$, and hence is not a part of $U$, which contradicts that everything is a part of $U$. So, if some possible world is junky, then universalism is not necessarily true. Q.E.D. ([3, p. 297, fn I ])

Now, the principle used in the argument is admittedly fairly plausible. Indeed, Bohn regards the weak supplementation principle as analytic of proper parthood. 
I assume at least some mereological principles without existential import are analytically true, and in virtue of that are necessarily true too. [...] Minimal Extensional Mereology is a minimum of mereological necessary truths. This system includes (among other things) the asymmetry and transitivity of proper parthood, as well as a weak supplementation principle. ([I], 29)

Plausible, but far from uncontroversial. I do not think weak supplementation is analytic, but I appreciate more needs to be said. ${ }^{4}$ In fact, as we will see below, there is a growing number of mereologists who reject weak supplementation. Some reject weak supplementation on its own terms, finding it independently objectionable. ${ }^{5}$ Others reject weak supplementation because they reject either irreflexivity ${ }^{6}$ and asymmetry ${ }^{7}$, which (in combination with transitivity) can be derived from weak supplementation.

Transitivity $(x<y \wedge y<z) \rightarrow x<z$

Irreflexivity $x \nless x$

Asymmetry $x<y \rightarrow y \nless x$

Many of the reasons given for rejecting irreflexivity and asymmetry concern exotic counterexamples, of which one might be suspicious. But there is a more philosophically robust reason enough to generalise the junk argument: it turns out that these 'minimal' principles are sufficient, when combined with unrestricted composition, to yield perhaps the most controversial mereological principle of all - extensionality, which says that composite objects with the same proper parts are identical. ${ }^{8}$ Extensionality forces us to identify objects, like perhaps the statue and the clay it is made from, which have all their parts in common. Given the indiscernibility of identicals, this

\footnotetext{
${ }^{4}$ For some considerations in this direction, see Cotnoir [8].

${ }^{5}$ Proponents include: Donnelly [I 2], Forrest [1 3], and Smith [27]. Also see Caplan et. al. [5] who express sympathy for the view.

${ }^{6}$ Proponents of dropping irreflexivity include Cotnoir and Bacon [10], Kearns [16], and Kleinschmidt [I7].

${ }^{7}$ Proponents of dropping asymmetry (or antisymmetry for parthood) for extensionality-independent reasons include Cotnoir and Bacon [Io], Kleinschmidt [17], Sanford [23], and Tillman and Fowler [3 I].

${ }^{8}$ See Varzi [35] to get a flavour of the debate.
} 
entails that any two composed objects with all the same proper parts have all the same properties. And whether e.g. the statue and the clay are indiscernible is a matter of serious contention.

The argument that universalists who accept weak supplementation and transitivity are committed to extensionality is due to Varzi [36]. Informally, imagine you had a counterexample to extensionality; suppose a statue $s$ and its clay $c$ are distinct objects with the same proper parts. By asymmetry, $s \nless c$, and $c \nless s .{ }^{9}$ By unrestricted fusion, there must be a sum $s+c$. (Note: $s+c \neq s$ since $c \leq s+c$ but $c \not \leq s$; likewise $s+c \neq c$, since $s \leq s+c$ but $s \not \leq c)$. Now, $s<s+c$; however, there is no proper part of $s+c$ that is disjoint from $s$, as any proper part of $c$ is also a proper part of $s$ by supposition. This violates weak supplementation. Hence, there can be no such counterexample to extensionality.

There are two ways to avoid Varzi's argument; as a result, there are two main approaches to mereology without extensionality: the unsupplemented view, ${ }^{10}$ and the mutual parts view. ${ }^{11}$ Both of these approaches are compatible with unrestricted composition; indeed, many proponents of these views themselves accept it. Since each explicitly rejects Bohn's assumption of weak supplementation, Bohn's defence of premise (i) is not available to them. But there are defences of premise (i) that non-classical mereologists can (and should) accept. I provide such defences in the next two sections for virtually any mereology with virtually any conception of unrestricted fusion.

\section{Weak Supplementation \& Junk}

The first main non-extensionalist option is the unsupplemented approach which drops the weak supplementation axiom above, but adds asymmetry. The unsupplemented approach has access to a well-developed mereology that includes unrestricted fusion. Here is a candidate list of axioms.

Asymmetry $x<y \rightarrow y \nless x$

\footnotetext{
${ }^{9}$ For, suppose $c<s$. Asymmetry implies $s \nless c$. Hence, $s$ and $c$ are not a counterexample to extensionality. Mutatis mutandis for the supposition that $s<c$.

${ }^{10}$ For extensionality-based reasons to drop weak supplementation, see Simons [26] and Gilmore[I4].

${ }^{11}$ For reasons to drop asymmetry to secure anti-extensionalism, see Thomson $[30,29]$ and Cotnoir $[7]$.
} 
Transitivity $(x<y \wedge y<z) \rightarrow x<z$

Unrestricted Composition $\forall x x \exists y \mathrm{~F}(y, x x)$.

Fusions appearing in the unrestricted composition axiom are defined as follows.

Fusion $\mathrm{F}(t, x x)$ iff $x x \leq t \wedge \forall y(y \leq t \rightarrow y \circ x x))$

(I write ' $x x \leq t$ ' to mean that every $x$ that is among $x x$ is part of $t$, and ' $y \circ x x$ ' to mean that $y$ overlaps some $x$ among the $x x$.)

On this view, proper parthood is a strict partial order; the major change is that weak supplementation is explicitly rejected. Some unsupplemented mereologists simply drop the axiom and leave it at that. ${ }^{12}$ Others (primarily Gilmore [I4]) have suggested a replacement axiom to capture the intuition behind weak supplementation without risk of extensionality. ${ }^{13}$ But as Bohn's defence of premise (i) explicitly appeals to weak supplementation, this defense is not available to unsupplemented anti-extensionalists, many of whom do accept unrestricted composition.

There is, however, a straightforward argument from transitivity and asymmetry (in place of weak supplementation) that unrestricted composition entails the negation of junk. Let $x x$ range over absolutely everything in a world. Since composition is unrestricted, there is some $u$ such that $\mathrm{F}(u, x x)$. As such, we have $x x \leq u$ (by the definition of fusion). Now, to satisfy the definition of junk, suppose $u<\hat{u}$. By asymmetry, $\hat{u} \nless u$, and thus $\hat{u} \not \leq u$. But that contradicts the supposition that $x x$ contains absolutely everything. So, there is no such $\hat{u}$, and hence the world is not junky.

None of this depends on any supplementation whatsoever. As a result, a version of the junk argument applies to unsupplemented anti-extensionalism.

\footnotetext{
${ }^{12}$ See the proponents listed in footnote 4 for the case for conceivability. It is also clear the approach is consistent (the axioms are satisfied in any complete lattice; for quasi-supplementation, consider complete join semi-lattices with at least two distinct minimal elements). So by Bohn's own criteria, the approach would appear to be metaphysically possible. It should at least be regarded as an available position in logical space.

${ }^{13}$ The relevant axiom is:

Quasi-Supplementation $x<y \rightarrow \exists w \exists z(z \leq y \wedge w \leq y \wedge \neg z \circ w)$
}

However this addition is strictly optional; there is a defense of premise (i) that goes through without it. 
Now, there is some debate over the correct notion of fusion for anti-extensionalists. In fact, some have held that the definition above is inadequate. ${ }^{14}$ However, it is worth noting that the above argument only relies on the fact that the fusion of $x x$ has each of the $x x$ as a part. Surely, any adequate notion of mereological fusion must be such that it is an upper bound in this sense of the things it fuses (even if not a least upper bound). That is, one cannot think $t$ is the fusion of the cats if some cat fails to be part of $t$. But then the above argument applies to unrestricted fusion of any sort.

\section{Asymmetry \&ojunk}

The second option for anti-extensionalists is the mutual parts view which rejects asymmetry for proper parthood. This allows for two distinct objects, such as the statue and the clay, to be parts of each other. As it turns out, this is sufficient for avoiding extensionality principles of all sorts. ${ }^{15}$ This approach has a growing number of advocates. ${ }^{16}$

The following axioms give a precise characterisation of the mutual parts approach.

Transitivity $(x<y \wedge y<z) \rightarrow x<z$

Remainder $y \not \leq x \rightarrow \exists z \forall w(w \leq z \leftrightarrow(w \leq y \wedge \neg w \circ x))$

\section{Unrestricted Composition $\forall x x \exists y \mathrm{~F}(y, x x) .{ }^{17}$}

As above, weak supplementation entails asymmetry, and so the mutual parts view must reject weak supplementation. However, the mutual parts view has access to a variety of very strong sup-

\footnotetext{
${ }^{14}$ See for example the arguments in Varzi [36] and Cotnoir [9].

${ }^{15}$ See Cotnoir [7] for an examination of the relation between antisymmetry and extensionality.

${ }^{16}$ Proponents of dropping antisymmetry (listed in footnote 5) have access to a well-developed mereology (which includes unrestricted fusion) with a class of well-defined models (see $[7, \mathrm{Io}]$ for details). Thus, the approach is consistent. It appears the approach satisfies Bohn's own criteria for possibility. At least very least, then, dropping asymmetry should be worthy of serious consideration.

${ }^{17} \mathrm{~A}$ variant weaker definition of fusion is typically used here:

$$
\mathrm{F}(t, x x) \text { iff } x x \leq t \wedge \forall y(x x \leq y \rightarrow t \leq y)
$$

However, the weakness of this definition is made up for by the corresponding strength of the remainder principle. (See [ Io], $\$ 3.2$ for more details).
} 
plementation principles. The strongest of these is the remainder principle, which says that if $y$ fails to be part of $x$, then there is an object composed of all and only the non-x-overlapping parts of $y$ - $x$ 's complement in $y .{ }^{18}$ It is commonly thought that strong supplementation principles like the remainder principle entail weak supplementation; but the proof of this fact relies on asymmetry. So the mutual parts view can accept such strong principles without falling into extensionality. ${ }^{19}$ This principle is again optional; however, we will return to this principle below $(\$ 5)$. But even in the presence of unrestricted fusion, this approach does not fall prey to the junk argument. There are models of this mereology that also satisfy Bohn's definition of junk, stating that everything is a proper part.

One such example is given by considering an $a$ and $b$ that are mutual parts such that everything else in the world is part of both. Such a world is consistent with unrestricted fusion, since $a$ and $b$ both count as fusions of the other. As is evident, $a$ and $b$ are distinct, so in fact there are two universal objects. Now, is this a junky world? In a sense, yes, since $a<b$, $a$ has everything as a proper part, but is itself a proper part of something (namely, of $b$ ). Likewise, since $b<a, b$ is has everything as a part but is itself a proper part of something (namely, $a$ ). So, according to the letter of the current definition of junk, premise (i) is strictly speaking false.

However, the kind of 'junk' displayed above is not the sort of junk one has in mind when levelling the argument against opponents of unrestricted composition. Proponents of junk are arguing for the existence of certain metaphysical structures; they are not (at least not primarily) arguing for the existence of worlds that satisfy a particular formal definition. As is often the case when one moves between formal systems, sometimes definitions that previously captured the intended structures fail to do so in a certain setting. ${ }^{20}$ Which definition captures the intended junky structures in the non-asymmetric setting? I contend that it is junk*:

Junk* $\forall x \exists y(x<y \wedge y \not \leq x)$

\footnotetext{
${ }^{18}$ Simons [26, p. 89] is responsible for the name. Varzi [34] and Hovda [15] call it Complementation.

${ }^{19}$ For more details, see Cotnoir [7].

${ }^{20}$ Compare the discussion regarding the correct definition of fusion (Varzi [36] and Cotnoir [9]) or the correct definition of proper parthood (Cotnoir [7], and Rea [22]) in non-extensional settings.
} 
So, a junky* world is a world in which everything is a proper part of something that is not also one of its own parts. In other words, junk* guarantees that everything is a non-mutual proper part (i.e. not a proper part of one of its proper parts, nor a proper part of itself). This sort of junk is incompatible with the above mereology. If $u$ is the fusion of everything $x x$, then $x x \leq u$. Now, to satisfy the definition of junk* , suppose $u<\hat{u}$ and $\hat{u} \not \leq u$. Contradiction.

This shows that premise (i) of the junk argument (on the intended reading, anyway) does not rely on any assumption of asymmetry.

\section{Binary Sums \& Junk}

I have been using 'Universalism' in the usual way to mean that whenever you have some things, there is a fusion of them. Contessa [6] distinguishes between strong and weak versions of universalism.

Strong Universalism For any plurality $x x$, there is an object that is their fusion. ${ }^{21}$

Weak Universalism For any pair of objects $x$ and $y$, there is an object that is their fusion.

Contessa contends that those who accept universalism should accept only the weak version and reject the strong. So for him, mereological universalists ought to accept only the existence of binary sums, and reject infinitary fusions.

To be clear about the context of this response, it is important to note that the junk argument was clearly originally intended as an objection to the strong version of the thesis. In fact, no one who calls herself a universalist accepts the weak thesis but not the strong. So, a natural reply springs to mind: the weak thesis isn't really a form of mereological universalism at all, is it? Of course it is compatible with universalism, as anyone who accepts the strong thesis would also

\footnotetext{
${ }^{21}$ It should be noted that Bohn [I] states the thesis as follows: "That is, assume that necessarily, any collection of things composes something" (p. 27). Likewise, Contessa states the strong thesis as follows: "for any collection of objects, there is always an object that is their mereological sum" (p. 456). But, given the connection between the notions of collection and set, we should be clear that it is only non-empty collections that have fusions; virtually no universalist thinks that the empty set has a fusion.
} 
accept the weak. But insofar as one accepts the weak thesis while rejecting the strong thesis, that view rejects the existence of certain kinds of fusions - it accepts that composition holds only in some cases (the finitary ones) and rejects that it holds under all cases (which would include the infinitary ones). For what it is worth, when Bohn [I] originally gives the junk argument, he considers such a view calling it explicitly a 'restricted view of composition'.

Contessa considers this thought and writes,

However, according to weak mereological universalism, that should not be understood as a restriction on composition, for, according to weak mereological universalism, mereological composition would be restricted only if there were pairs of objects that, under some circumstances, did not have a mereological sum and, since junky worlds do not feature any such pair of objects, they do not constitute a counterexample to weak mereological universalism. ([6], p. 456)

It is not clear from the passage precisely what Contessa has in mind here as a dialectically effective reply. The statement that "composition would be restricted only if there were pairs of objects that $[\ldots]$ did not have a mereological sum" suggests the idea that composition is fundamentally a binary operation, not properly thought of as an operation on collections. ${ }^{22}$ A related thought is that composition is essentially binary - the composition operation by its very nature takes two objects as inputs and outputs some further object. On that understanding of composition, weak universalists do think composition is unrestricted. Infinitary cases of compositions are no counterexample to universalism because infinitary collections of objects aren't even 'composition$a p t^{\prime}$. Composition doesn't fail to obtain, it fails to apply.

There are a number of reasons one might find this strict version of weak universalism unsatisfying. Let me first consider a bad reason for rejecting it before going on to present my own preferred reasons. Bohn [I] objects to this view by arguing that it is incompatible with the existence of gunky worlds — worlds in which everything has a proper part. Bohn claims:

\footnotetext{
${ }^{22}$ Contessa has confirmed in correspondence that this is in fact the view he had in mind.
} 
That means that if the world is finite, it contains a universal object $U$, while if the world is infinite with mereological atoms, it is junky. The problem with this principle is that it is incompatible with the world being gunky. A gunky world is such that everything in it has a proper part. If everything in the world has a proper part, then every fusion in the world is infinitely divisible, which means that every fusion in the world is of infinite cardinality, which again means that no fusion in the world is of finite cardinality. Since our principle implies that necessarily all fusions are of finite cardinality, it must therefore be incompatible with the possibility of gunk. ([I, p. 30], emphasis mine)

There two things to say about this argument. First, it is true that a gunky object $a$ would be a case of infinitary fusion. Let $a a$ be the plurality of all the proper parts of the gunky object $a$. Then $a a$ is an infinite collection such that $F(a, a a)$. Must the weak universalist reject the existence of such an object? I think the answer is 'no'. A weak universalist might accept the existence of $a$ so long as there exist some proper parts $a_{1}$ and $a_{2}$, such that their sum just is $a$. The problem with Bohn's argument is with the emphasised portion of the passage. Just because the world is infinite (and indeed, every object infinitely divisible), it does not follow that every fusion must be infinite. While gunky objects have infinitely descending proper parthood chains, the key issue is whether there are infinitely ascending chains leading up to the gunky object. The weak universalist might accept the possible existence of objects with infinitely many proper parts, so long as we can always fuse our way up to it in finitely-many steps. ${ }^{23}$

So Bohn's objection to the weak thesis fails. However, there is another argument against this particular essentialist version of weak universalism: it is in tension with an important mereological principle — the remainder principle above. Recall:

\footnotetext{
${ }^{23}$ Consider a non-junky but gunky model $M$; in particular, suppose $M$ has no infinite ascending <-chains, but has infinite descending <-chains. It follows that for every non-empty subset $A$ of $M$, there is some finite subset $F$ of $A$ such that the least upper bound of $F$ is the least upper bound of $A$. (For a proof of this fact, see Davey and Priestly [i I, p. 52], Theorem 2.4I (i).) Since we can take all the members of $F$ and fuse them pair-wise, it follows that every non-empty subset $A$ has a fusion.
} 
Remainder $y \not \leq x \rightarrow \exists z \forall w(w \leq z \leftrightarrow(w \leq y \wedge \neg w \circ x))$

The principle entails that where $y$ is has a proper part $x$, there must be a 'remainder' or 'complement' of $x$ in $y$. The remainder $z$ has as parts all and only the non-x-overlapping parts of $y$. But the non- $x$-overlapping parts of $y$ might well be infinite. And it turns out (by the definition of fusion) that $z$ is the fusion of precisely these parts. To see this, fix some particular $x$ and $y$, such that $z$ is this remainder. Let $w w$ be the plurality of objects satisfying the open sentence ' $(w \leq y \wedge \neg w \circ x)$ '. To establish that $F(z, w w)$ we need to show that $w w \leq z$ and $\forall w(w \leq z \rightarrow w \circ w w))$. The former we have by the right-to-left direction of the consequent of the remainder principle. For the latter, suppose $w \leq z$. Then by the left-to-right direction of the consequent, we have it that $w$ is such that $(w \leq y \wedge \neg w \circ x))$, which means by definition it is identical to one of the $w w$, and hence overlaps one of them. Thus the remainder principle entails that $z$ is the fusion of $w w$.

The weak universalist cannot simply stipulate the remainder principle as an axiom without running the risk of thereby accepting infinitary fusions. Now it may well be in some cases (as in the case of gunk without infinite ascending <-chains above), that $z$ can be constructed pair-wise from other objects. But the weak universalist who rejects purely infinite fusions needs it to be the case that every remainder can be constructed in this way from finite sums. However, there is no general guarantee that this is the case; there is nothing in the axioms of her mereology that rule out structures for which remainders cannot be so constructed. Problematic cases involve worlds with infinite ascending proper parthood chains up to some composed whole - call them nonNoetherian worlds. ${ }^{24}$ Such worlds come in a number of varieties: worlds which are both junky and gunky (i.e. what Bohn [2] calls 'hunky') can be of this type, as are worlds where the proper parthood chain is dense, i.e. for every $x$ and $z$ such that $x<z$, there is some $y$ such that $x<y<z$.

For a concrete example of the latter, suppose we have an object $q$ be the interval of rational numbers between 0 and 1 (inclusive). Now focus on the 'right half' $r$ of $q$, namely the interval of

\footnotetext{
${ }^{24}$ Let $M^{\prime}$ be a model of a non-Noetherian world. Since such a world has infinite ascending <-chains, we can no longer prove that every non-empty subset $A$ of $M^{\prime}$ has a corresponding finite subset $F$ such that the least upper bound of $F$ is the least upper bound $A$. This may be (in the case of junk) because $A$ has no upper bound at all in $M^{\prime}$, or it may be that even though $A$ has a least upper bound, the only way to fuse to $A$ is by taking the fusion of all of its members.
} 
numbers between and including $\frac{1}{2}$ and 1 . Since $[0,1] \not \leq\left[\frac{1}{2}, 1\right]$, by the remainder principle, there must be some object $l$ (the 'left half') composed of all and only those parts of $[0,1]$ that do not overlap $\left[\frac{1}{2}, 1\right]$. So it must contain every number starting from 0 and up to (but not including) $\frac{1}{2}$, namely the interval $\left[0, \frac{1}{2}\right)$. Notice, because of the density of the rationals, there is no last number in this interval. So if we are trying to generate it by composing it pairwise with other rational numbers, we will fail. For every object we sum up, there will always be infinitely many more. We would have a finite sum up to $r$ if there were certain open intervals around, but these are precisely what we don't know to exist. And it's not clear there is any way of ensuring they exist without also committing one to infinitary fusions.

There are a few avenues of reply: one might (a) reject the remainder principle, (b) reject the existence of non-Noetherian worlds (i.e. reject the possibility of hunky worlds or dense worlds), or (c) accept both, and allow some cases of infinitary composition. Against (a), I would urge that the remainder principle is an important part of classical mereology; it is what makes mereology a Boolean algebra after all. The resulting mereology would be highly non-classical in ways unrelated to the existence of junk. ${ }^{25}$ Against (b), it would be very odd (even if not incoherent) if in defending the possibility of infinite ascent of one type — namely, junky worlds - one was forced into denying the possibility of infinite ascent of another type — namely, hunky or dense worlds. Why should it be the case that any world with proper parthood chains that continue infinitely upward cannot have any proper parthood chains that also continue infinitely up to a whole? Absent some principled metaphysical reason, it is a dialectically weak position to find oneself defending, at any rate. ${ }^{26}$ Moreover, given that many philosophers think that regions of space are continuous, and hence dense, option (b) rules out a very common view about the structure of the actual world.

So I think the best option is (c). In fact, this is the view of the only mereologists (that I know of) who accept the weak thesis but not the strong thesis. For example, Bostock’s [4, ch. $2 \$ 4]$ mereology was devised for the foundations of arithmetic in the rational numbers; as such, his

\footnotetext{
${ }^{25}$ For more discussion, see Hovda [I 5 ], especially footnote 25.

${ }^{26}$ See also the arguments in Bohn $\left[2, \S_{2}\right]$.
} 
mereology is quite robust. It allows for gunk and junk and dense proper parthood chains, and also satisfies the remainder principle. The main departure from classical mereology that Bostock proposes is a variant fusion axiom, which (in our notation) is:

Moderately Unrestricted Composition $\forall x x(\exists y(x x \leq y) \rightarrow \exists z \mathrm{~F}(z, x x))^{27}$

What this principle says is that if $x x$ have an upper bound, then they must have a fusion. Of course, this entails that if some infinite collection is bounded from above, then it must have a fusion. This is compatible with the existence of junk, though, since not all infinite collections are required to be bounded from above. However, it is not compatible with the strict essentialist version weak universalist thesis, since it entails that some infinite collections have fusions. ${ }^{28}$

Moreoever, 'moderately unrestricted' composition makes it plain that the view is not really a version of mereological universalism at all. The fusion axiom is plainly restricted by the antecedent - only collections that are bounded have fusions. And so, I view this response as changing the terms of the debate. It may well be the most plausible version of a junky mereology, but it hardly constitutes a counterinstance to premise (i).

\section{$5 \quad$ Unrestricted Quantification \& Junk}

Spencer [28] has recently argued against premise (i) due to the fact that its defence relies on unrestricted plural quantification. ${ }^{29}$ That is, the incompatibility of junk and unrestricted composition requires the supposition that there are some things $x x$ — call them 'all things' — such that everything is amongst them.

\footnotetext{
${ }^{27}$ The definition of fusion is different from above. For Bostock [4, p. I I 8], fusion is defined thus: $\mathrm{F}^{\prime}(t, x x)$ iff $\forall y(y \circ t \leftrightarrow y \circ x x)$, although in the presence of the remainder principle, the two definitions are equivalent.

${ }^{28}$ For a sophisticated discussion of related issues, see Linnebo, Hellman, and Shapiro's [20] mereological conception of the Aristotelian continuum. They openly admit that their view is not a universalist view (as such, none of the arguments of this paper are aimed at them), but do wish to avoid actual infinities insofar as it is possible. The main difficulties involve securing the existence of differences (i.e. remainders), bisections, and biextensions, as well as proving the Archimedian principle. They propose a number of different fusion axioms to attempt to deal with the difficulties.

${ }^{29}$ This is related to Bohn's explicit claim that "Universalism [is defined as] $\forall x x \exists y(x x$ compose $y)$, where the quantifiers are unrestricted and ' $x x^{\prime}$ ' is a plural variable taking one or more things as its value" ([3, p. 297]).
} 
[T]his argument clearly relies on the premise that there are some things that all things are amongst. If there are no such things, then the argument is unsound. In fact, unrestricted composition and [the denial of 'all things'] together entail junk. ([28, p. 7I])

While this approach is the best attempt at avoiding premise (i), it is less than clear that the mere denial of 'all things' will deliver the compossibility of unrestricted composition and junky worlds in the required sense.

Before delving into Spencer's arguments, we need to be clear on what it is that Spencer is rejecting. We need to distinguish between (a) absolutely unrestricted singular quantification and absolutely unrestricted plural quantification, and (b) two different readings of what it is for a plural quantifier to be absolutely unrestricted.

For (a), there is an important difference between absolutely unrestricted singular quantification and absolutely unrestricted plural quantification. The former is used in the definition of junk, while the latter is used in the fusion axiom. Spencer is clear that he is not denying that the singular universal quantifier is absolutely unrestricted. But one might deny this, and we should consider whether that might affect premise (i) of the junk argument. So let us suppose that our singular universal quantifier fails to be unrestricted. Notice that junky worlds are themselves defined using the universal quantifier. So, if our universal quantifier fails to quantify over everything, it may well be that those objects not in its scope will fail to have a fusion. However, even if so it will not follow that the world satisfies the definition of being junky, since it will not follow that $\forall x \exists y(x<y)$ is true. Why? Because those objects that fall outside the scope of the unrestricted fusion axiom will also fall outside the scope of the quantifier in the statement of junk. All that could be maintained in this case, is the might be worlds that are inexpressibly junky. But the metaphysical possibility of inexpressibly junky worlds has not been argued for. It needs an independent defence — a defence that goes well beyond the defence of premise (ii). 
Returning to (b), we need to clarify what is at issue in claiming that a plural quantifier is unrestricted. There are two possible ways of thinking about this. First, we might think a plural quantifier is unrestricted if and only if it ranges over absolutely everything; that is, for every thing $x$, there is some plurality $x x$ that $x$ is among. On this reading, Spencer is definitely not arguing against unrestricted plural quantification.

However, there is a second way of thinking about what it is for a plural quantifier to be unrestricted: it is unrestricted if and only if it ranges over absolutely all pluralities, where pluralities are generated from all the instances of plural comprehension. On this reading, Spencer is arguing against unrestricted plural quantification, since he rejects that there is a plurality of all things. To be precise, Spencer expresses the denial of 'all things' thus: $\neg \exists x x \forall y y(y y$ are among $x x)$. He has to deny plural comprehension, since were $z z$ to contain all and only the objects satisfying the predicate $\exists x(z=x)$, it would consist of 'all things'.

Now we are in a position to consider Spencer's argument against premise (i). ${ }^{30}$ Spencer's argument purports to show that a world in which universalism is true and without 'all things' must be a junky world.

Consider some arbitrary thing 'Chunk' and call all of its parts 'Bits'. Now if [the existence of 'all things'] is false, there are some things that Chunk's parts are properly amongst. Call those things 'Bits+Pieces'. Now, there are some things amongst Bits+Pieces that are not parts of Chunk [...] [namely,] Pieces. According to unrestricted composition there is something composed of Chunk and Pieces, namely Big Chunk. Moreover since Big Chunk has parts that are not parts of Chunk, namely Pieces, it follows that Chunk is a proper part of Big Chunk. So, there is something that Chunk is a proper part of. But, since Chunk was arbitrarily chosen, it follows that for anything whatsoever, there is something that it is a proper part of. So Junk is true. Again, unrestricted composition and [the denial of 'all things'] entails Junk. ( [28, p. 72])

\footnotetext{
${ }^{30}$ I focus only on his second (more general) argument. What I say about the second applies equally to the first.
} 
The argument has a number of moving parts; however, we do not have to read very far to find some potentially objectionable assumptions being made. The emphasised passage contains two such assumptions. The first is that for any arbitrary thing, there is a plurality of all its parts (in this case, 'Bits'). The second assumption claims that if there is no plurality of all things, then there must be a plurality that properly contains all of the parts of a given thing (in this case, 'Bits+Pieces'). But why think either assumption is true? I will consider each in turn.

Regarding the first assumption, here is a possible scenario. Suppose we can quantify over every macro-level object but we cannot quantify over all their parts. It may well be that the world gets 'too numerous' as we move to more and more finely grained levels of reality. We may be simply unable to quantify over all things because we cannot quantify over all the parts of things.

If we can quantify over objects at some macro-level specification, by unrestricted fusion (of any sort), we will have it that every parthood chain has an upper bound. Hence, we know that the world has at least one <-maximal element (an element with nothing containing it as a proper part). ${ }^{31}$ But a maximal element entails the failure of junk. For a concrete example, imagine the domain of all subsets of the $[0,1]$ interval of the real numbers. Imagine that pluralities can take at most countably many subsets of that interval. Let $y y$ be the plurality containing two intervals: $\left[0, \frac{1}{2}\right]$ and $\left[\frac{1}{2}, 1\right]$. The fusion of $y y$ is the whole unit, even though there is no plurality of all subsets of the unit.

It is worth noting that Spencer recognises that his argument relies on this assumption, and suggests that its failure would be an "interesting metaphysical result" (72). Still, such a possibility shows that a universalist who rejects unrestricted plural quantification is not committed to junk. What is required, then, is an argument for thinking that our quantifier fails to quantify over absolutely everything going upwards along parthood chains, rather than the failure occurring as

${ }^{31}$ This requires an application of Zorn's Lemma, which is equivalent to the Axiom of Choice.

Zorn's Lemma Let $M$ be any non-empty partially ordered set. If every chain has an upper bound, then $M$ has a maximal element.

Suppose $M$ here is a model of the mereological structure of the world, and the partial order on $M$ is just the parthood relation. A chain is defined to be a totally ordered subset of $M$; so, a subset such that for every $x$ and $y$, either $x \leq y$ or $y \leq x$. 
we go downwards. What we need in short is an argument in favour of premise (ii) of the junk argument.

There is a second assumption in Spencer's argument: the denial of all things entails that for any plurality, there must be another plurality that properly contains it. Presumably, what Spencer is thinking is this: suppose $a a$ are some things and that there is no plurality that properly contains $a a$. Then $a a$ would be a plurality of everything, which we are supposing does not exist. But this does not follow, as a might just be the largest plurality we can quantify over. As such, the assumption is entirely unwarranted in the context.

To make the matter concrete, let us give a metaphysical example. Suppose there are too many mereological simples to quantify over. In that case, we couldn't quantify over all things because we couldn't quantify over all the smallest things. If there are too many simples to form a plurality, those simples might not be parts of anything due to the corresponding expressive weakness of the unrestricted composition axiom. Still, each individual simple will be an upper bound of the (trivial) chain containing only itself. Every other chain will have an upper bound by unrestricted fusion. Hence, we will have a maximal element of the world — in this case, perhaps lots! ${ }^{32}$ But the existence of such maximal elements means that the world cannot be a junky world. Note well: this is all perfectly compatible with the failure of the universal quantifier to be absolutely general.

Again what are not given, and what is required for the argument, is a reason to think that for any plurality, there is always a more expansive plurality containing it, i.e. $\forall x x \exists y y(x x$ are properly among $y y)$. This is strikingly close to the assumption of junk, i.e. $\forall x \exists y(x<y) .{ }^{33}$ The best way forward, I think, would motivate the failure of unrestricted plural quantification via indefinite extensibility. That is, whenever we have 'all things' at one level, we can construct some new thing which has all previous things as proper parts. So, at each level $n$ in the construction, there is some further thing at level $n+1$ which failed to be quantified over at level $n$. This typed approach is seen clearly by

\footnotetext{
${ }^{32}$ Again, this follows by an application of Zorn's Lemma.

${ }^{33}$ Where $\preceq$ is the symbol for the plural 'are among' relation, the denial of 'all things' is logically equivalent to $\forall x x \exists y(y y \npreceq x x)$. This is structurally analogous to something entailed by the definition of junk, but which does not itself entail junk: namely $\forall x \exists y(y \not \leq x)$.
} 
indexing our quantifiers. Thus, a kind of 'junk' becomes expressible:

Typed Junk $\forall_{n} x \exists_{n+1} y(x<y)$

Likewise, the corresponding version of 'unrestricted' composition becomes:

Typed Composition $\forall_{n} x x \exists_{n+1} y \mathrm{~F}(y, x x)$

On this view, junk is compatible with typed composition. However, one should immediately begin to wonder whether this typed version of composition is really unrestricted after all. How are we supposed to admit that our quantifiers fail to be unrestricted, and yet accept that composition is unrestricted if they exhibit the very same phenomena? We might say that junk is still incompatible with absolutely unrestricted composition; and reword premise (i) accordingly.

Of course, the reply will come: but there is no such thing as absolutely unrestricted composition - some variant of our typed composition is the best anyone can do! I am not sure how to adjudicate this debate over whether something counts as 'unrestricted' or not. The issues are too complex to delve into here. But I'll just note that if the reply is correct, then it depends on the arguments for the metaphysical possibility of indefinite extensibility, and the corresponding sort of junk generated from it. And so it still seems to me to boil down to a case of requiring a robust defence of premise (ii).

\section{Concluding Remarks}

I have argued that the junk argument can be modified to apply to virtually any mereology extensional or not - that accepts unrestricted composition of any sort. I've shown that the retreat the the weak version of mereological universalism is not a promising move. It either requires more widescale revisions to mereological structure, or is explicitly a form of restricted composition. Moreover, I have argued that rejecting absolutely general quantification does not (at least by itself) ensure the possibility junky worlds. One might allow for inexpressible gunk, but this option goes well beyond anything that premise (ii) provides. Or if one expresses junk 
and composition via type restrictions, then it's unclear that the view really does represent a form of unrestricted mereological composition. I conclude that premise (i) of the junk argument is undefeated. The case against universalism rests entirely on premise (ii). ${ }^{34}$

University of St Andrews St Andrews, Fife Ky I 6 9AR, uK acII7@st-andrews.ac.uk

\section{References}

[I] Bohn, E. An argument against the necessity of unrestricted composition. Analysis 69, I (2009), 27-3I.

[2] Bohn, E. Must there be a top level? The Philosophical Quarterly 59 (2009), I93-20 I.

[3] BoHn, E. The necessity of universalism vs. the possibility of junky worlds: a rejoinder. Analysis 70 (2010), 296-298.

[4] Bозтоск, D. Logic and Arithmentic: Rational and Irrational Numbers, vol. 2. Clarendon Press, I979.

[5] Caplan, B., Tillman, C., and Reeder, P. Parts of singletons. Journal of Philosophy io7 (2010), 50I-533.

[6] Contessa, G. The junk argument: Safe disposal guidelines for mereological universalists. Analysis 72 (201 2), 455-457.

[7] Cotnoir, A. J. Anti-symmetry and non-extensional mereology. The Philosophical Quarterly 60,239 (2010), 396-405.

[8] Cotnoir, A. J. Strange parts: the metaphysics of non-classical mereologies. Philosophy Compass 8, 9 (2013), 834-845.

[9] Cotnorr, A. J. Does universalism entail extensionalism? Nô̂s EarlyView (20 I 4).

\footnotetext{
${ }^{34}$ Thanks to Einar Bohn, Gabriele Contessa, Janelle Derstine, and Stewart Shapiro for comments on earlier drafts. This paper was presented at the PERSP metaphysics seminar at the University of Barcelona, the MmM Arché seminar at the University of St Andrews, and the Philosophy Society Colloquium at Oxford University. Thanks to all the attendees for great discussions, but particular thanks are due to Frank Arntzenius, John Hawthorne, Bruno Jacinto, Spencer Johnston, Martin Lipman, Dan Lopez de Sa, Ofra Magidor, Giovanni Merlo, Josh Parsons, Bryan Pickel, Sven Rosenkrantz, Michael Traynor, and Richard Woodward. I also wish to thank the seminar participants at csmn in the University of Olso. Special thanks to an anonymous referee whose excellent comments led to many improvements in this paper.
} 
[i o] Cotnoir, A. J., And Bacon, A. Non-wellfounded mereology. Review of Symbolic Logic 5, 2 (2012), I 87-204.

[i i] Davey, B., and Priestry, H. A. Introduction to Lattices and Order, 2nd ed. Cambridge University Press, 2002.

[12] Donnelly, M. Using mereological principles to support metaphysics. The Philosophical Quarterly 6I (20II), 225-246.

[13] Forrest, P. Non-classical mereology and its application to sets. Notre Dame Journal of Formal Logic 43 (2002), 79-94.

[14] Gilmore, C. Quasi-supplementation, plenitudinous coincidentalism, and gunk. In Substance: New Essays. Philosophia Verlag, forthcoming.

[ I 5] Hovda, P. What is classical mereology? Journal of Philosophical Logic 38, I (2009), 55-82.

[16] Kearns, S. Can a thing be part of itself? American Philosophical Quarterly 48, I (20 I I).

[i7] Kleinschmidt, S. Multilocation and mereology. Philosophical Perspectives 25 (20 I I), 253276.

[18] Leibniz, G. New Essays on Human Understanding. Cambridge University Press, 1996.

[i9] Lewis, D. Parts of Classes. Basil Blackwell, Oxford, I991.

[20] Linnebo, O., Hellman, G., and Shapiro, S. An aristotelian continuum. ms.

[2 I] REA, M. In defense of mereological universalism. Philosophy and Phenomenological Research 58 (1998), 347-360.

[22] Rea, M. Universalism and extensionalism: a reply to Varzi. Analysis 70, 3 (20 10), 490-496.

[23] SANFord, D. The problem of the many, many composition questions, and naïve mereology. Noûs 27, 2 (June 1993), 219-228.

[24] Schaffer, J. Monism: The priority of the whole. Philosophical Review II9 (2010), 3 I-76.

[25] Sider, T. Parthood. The Philosophical Review II6 (2007), 5 I-9I.

[26] Simons, P. M. Parts: A Study In Ontology. Clarendon, Oxford, I 987.

[27] Smith, D. Mereology without weak supplementation. Australasian Journal of Philosophy 87 (2009), 505-5II.

[28] Spencer, J. All things must pass away. In Oxford Studies in Metaphysics, D. Zimmerman and K. Bennett, Eds., vol. 7. Oxford University Press, 20 I 2.

[29] Thomson, J. J. Parthood and identity across time. Journal of Philosophy 80, 4 (I983), $20 \mathrm{I}-220$. 
[30] Thomson, J. J. The statue and the clay. Nồs 32, 2 (June I998), I 49-I73.

[3 I] Tillman, C., and Fowler, G. Propositions and parthood: The universe and antisymmetry. Australasian Journal of Philosophy 90 (2012), 525-539.

[32] van Cleve, J. The moon and the sixpence: In defense of mereological universalism. In Contemporary Debates in Metaphysics, T. S. J. Hawthorne and D. Zimmerman, Eds. Blackwell, Oxford, 2008, pp. 22 I-240.

[33] VARZI, A. Mereological commitments. dialectica 54 (2000), 283-305.

[34] Varzi, A. Mereology. In The Stanford Encyclopedia of Philosophy, E. N. Zalta, Ed. 2006.

[35] VarzI, A. The extensionality of parthood and composition. The Philosophical Quarterly 58 (2008), I08-I33.

[36] VArZI, A. Universalism entails extensionalism. Analysis 69 (2009), 599-604.

[37] Watson, D. An argument against an argument against the necessity of universal mereological composition. Analysis 70 (2010), 78-82.

[38] Whitenead, A. An Enquiry Concerning The Principles Of Natural Knowledge. Cambridge University Press, I9I9. 